\title{
Disponibilidade de zinco para milho em resposta à localização de fósforo no solo
}

\author{
Lúcio H. De Muner' ${ }^{1}$, H ugo A. Ruiz², Victor H. A. Venegas², Júlio C. L. N eves², \\ Fernando J. Freire ${ }^{3} \&$ Maria B. G . dosS. Freire ${ }^{3}$
}

\begin{abstract}
RESUMO
A aplicação de P no solo pode afetar a disponibilidade de $\mathrm{Zn}$ para as plantas, dependendo de sua localização. Com o objetivo de avaliar a resposta de milho à adição de $\mathrm{Zn}$ no solo e determinar seu nível crítico no solo e na planta, em relação à localização de $\mathrm{P}$, em amostras de dois solos de Minas G erais, realizou-se um ensaio em casa de vegetação. O experimento consistiu em um arranjo fatorial $(2 \times 3 \times 6)$, correspondendo a dois solos (Latossolo Vermelho Amarelo variação U na e Argissolo Vermelho Amarelo, doravante denominados LU e PVA, respectivamente); três modos de localização de P $(5,10$ e $30 \%$ do volume total do solo na dose de $\left.200 \mathrm{mg} \mathrm{dm}^{-3}\right)$ e seis doses de $\mathrm{Zn}\left(0,1,2,4,6\right.$ e $\left.8 \mathrm{mg} \mathrm{dm}^{-3}\right)$. D eterminaramse a produção de matéria seca e os teores de $\mathrm{Zn}$ na parte aérea das plantas de milho. A adição de $\mathrm{Zn}$ aumentou linearmente $(p<0,01)$ o teor do elemento no solo e a absorção pela planta. No solo LU os níveis críticos variaram de 0,91 a 2,13 $\mathrm{mg} \mathrm{dm}^{-3}$ de $\mathrm{Zn}$ pelo extrator M ehlich-1 e, na planta, de 18,0 a $24,6 \mathrm{mg} \mathrm{kg}^{-1}$. Esses níveis foram maiores quando as aplicações de $\mathrm{P}$ foram local izadas em menor volume de solo. A localização de $P$ afetou o teor de $\mathrm{Zn}$ na Planta. No solo LU, quando o $\mathrm{P}$ foi localizado no menor volume de solo e na ausência de $\mathrm{Zn}$, as plantas de milho apresentaram elevados teores de $\mathrm{P}$.
\end{abstract}

Palavras-chave: nível crítico de Zn, forma de aplicação de P, interação Zn/P

\section{Availability of zinc to corn in response to liming and added phosphorus placement in soil}

\begin{abstract}
The soil $P$ application can affect $Z n$ availability to plants, depending on the application. The objective of this study was to evaluate corn (Zea mays L.) response to zinc (Zn) addition to the soil and to determine its critical level in the soil and in the plant, in relation to added phosphorus (P) placement, using two soil samples from Minas Gerais, Brazil, in a greenhouse experiment. The experiment was conducted in a factorial arrangement $(2 \times 3 \times 6)$, with two soils [Red Yellow Latosol of the Una Variation $(0 \times 1$ sol) and RedYellow Podzolic (UItisol), named LU and PVA, respectively]; three forms of added P placement (5, 10 and $30 \%$ of the total volume of soil in a dosage of $\left.200 \mathrm{mg} \mathrm{dm}^{-3}\right)$, and six $Z \mathrm{n}$ dosages $\left(0,1,2,4,6\right.$ and $8 \mathrm{mg} \mathrm{dm}^{-}$ $\left.{ }^{3}\right)$. The dry matter production and the contents of $\mathrm{Zn}$ in the aerial part of the corn plants were determined. The addition of $\mathrm{Zn}$ linearly increased $(\mathrm{p}<0.01)$ its content in the soil and plant uptake. The critical levels for the LU soil ranged from 0.91 to $2.13 \mathrm{mg} \mathrm{dm}^{-3}$ of $\mathrm{Zn}$ as determined by the Mehlich-1 extractor and plant levels ranged from 18.0 to $24.6 \mathrm{mg} \mathrm{kg}^{-1}$. These levels were higher when $\mathrm{P}$ was placed in smaller soil volumes. P placement affected the $\mathrm{Zn}$ content in the plant. In LU soil, when P was placed at the smallest soil volume and $\mathrm{Zn}$ privation, the corn plants presented elevated $\mathrm{P}$ levels.
\end{abstract}

Key words: Critical level of zinc, phosphorus placement, interaction Zn/P

IN CAPER/Instituto Capixaba de Pesquisa, Assistência Técnica e Extensão Rural, Rua Afonso Sarlo 160, Bento Ferreira, CEP 29052-010, Vitória, ES. Fone: (27) 3137-9888. E-mail:demuner@incaper.es.gov.br

2 Departamento de Solos/U FV, Av. Peter Henry Rolfs s/n, Campus U niversitário, CEP 36570-000, Viçosa, MG. Fone: (31) 3899-2200, Fax: (31) 3899-2108. E-mail: hruiz@ufv.br; vhav@ufv.br; Julionn2003@yahoo.com.br

3 D epartamento de Agronomia/U FRPE, Rua D om Manoē de M edeiros, Campus U niversitário de D ois Irmãos, 52171-900, Recife, PE. Fone: (81) 3320-6223, Fax: (81) 3320-6220. E-mail: f.freire@depa.ufrpe.br; betania@depa.ufrpe.br 


\section{INTRODUÇÃO}

Existe muita controvérsia quando se discute a interação $\mathrm{Zn} / \mathrm{P}$. Diversos autores afirmam que os problemas gerados pela interação ocorrem no solo onde a disponibilidade e a taxa de difusão de $\mathrm{Zn}$ são reduzidas por um elevado suprimento de $\mathrm{P}$ (Carneiro et al., 2008). Outros, no entanto, asseguram que estes ocorrem no interior da planta, alterando os processos metabólicos (Furlani et al., 2005).

Shuman (1988) sugeriu que altos teores de P podem afetar a disponibilidade de Zn. Quando o P é adicionado ao solo, pelo menos quatro processos poderiam modificar seu equilíbrio: mudanças no $\mathrm{pH}$, em virtude da dissolução do fertilizante na solução do solo; mudanças no pH, em consequência das reações, tanto do fosfato quanto do cátion acompanhante com os componentes do solo; mudanças na carga de superfície, em virtude da adsorção dos fosfatos pelos coloides e precipitação direta do $\mathrm{Zn}$ com o fosfato, formando $\mathrm{Zn}_{3}\left(\mathrm{PO}_{4}\right)_{2}$. Porém a ação depressiva da adubação fosfatada sobre a disponibilidade de Zn é pouco conhecida, visto não se conhecerem os compostos minerais que controlam a solubilidade do $\mathrm{Zn}$ no solo. As sugestões sobre sua precipitação na forma de $\mathrm{ZnCO}_{3}, \mathrm{Zn}(\mathrm{OH})_{2}$, $\mathrm{Zn}_{3}\left(\mathrm{PO}_{4}\right)_{2}, \mathrm{ZnSiO}_{3}$ e $\mathrm{ZnSO}_{4}$ parecem ser inconsistentes, uma vez que a concentração de equilíbrio do $\mathrm{Zn}^{2+}$ no solo é mais baixa que a fornecida por esses compostos, considerados boas fontes de Zn para o vegetal (Elrashidi \& O'Connor, 1982).

Estudando o efeito do $\mathrm{P}$ e do $\mathrm{Zn}$ sobre o crescimento e a nutrição de arroz em casa de vegetação, Haldar \& Mandal (1981) constataram que as aplicações de $\mathrm{P}$ e $\mathrm{Zn}$ aumentaram significativamente a produção de matéria seca da parte aérea e das raízes. As doses de 50 e $100 \mathrm{mg} \mathrm{dm}^{-3}$ de $\mathrm{P}$ causaram, porém, um decréscimo na concentração de $\mathrm{Zn}$, tanto na parte aérea quanto nas raízes. Da mesma forma, as aplicações de 5 e $10 \mathrm{mg}$ $\mathrm{dm}^{-3} \mathrm{de} \mathrm{Zn}$ diminuíram a concentração de $\mathrm{P}$ nesses tecidos. Tal decréscimo na concentração dos elementos não ocorreu em virtude do efeito de diluição ou da reduzida taxa de translocação das raízes para a parte aérea, mas das alterações na disponibilidade desses elementos no solo, pela aplicação conjunta de $\mathrm{P}$ e Zn.

Altas doses de $\mathrm{P}$ aplicadas em solos pobres em $\mathrm{Zn}$ podem induzir à deficiência de $\mathrm{Zn}$, em consequência da interação no solo e/ou, na planta (Robson \& Pitman, 1983). Citam-se a baixa velocidade de translocação do Zn das raízes para a parte aérea (Souza et al., 2006); o efeito de diluição do Zn na parte aérea, pelo crescimento da planta, em resposta à adição do $\mathrm{P}$ (Marinho \& Igue, 1972) ou o distúrbio metabólico nas células da planta, provoca-do pelo desequilíbrio entre P e Zn (Loneragan et al., 1982). Souza et al. (2006) constataram interação negativa entre $\mathrm{P}$ e $\mathrm{Zn}$ em mudas de cacaueiro, princi-palmente nas raízes, o que resultou na redução da translocação de $\mathrm{Zn}$ para as folhas.

Loneragan et al. (1982) relataram que a deficiência de Zn estimula não só a taxa de absorção de $\mathrm{P}$ pelas raízes como também a translocação de P para a parte aérea. Este estímulo é específico para o $\mathrm{Zn}$, não sendo verificado para outros micronutrientes.

Carneiro et al. (2008), estudando fontes, doses e modos de aplicação de $\mathrm{P}$ na interação $\mathrm{Zn} / \mathrm{P}$ em dois cultivos sucessivos de milho em Argissolo Vermelho típico de Cerrado, observaram que os teores de Zn nas plantas diminuíram com a aplicação de fonte solúvel de P no sulco de plantio sem, contudo, afetar significativamente a produtividade da cultura.

Assim, com o objetivo de avaliar a disponibilidade de Zn no solo, a resposta de milho à adição desse nutriente e as interações $\mathrm{Zn} / \mathrm{P}$ e suas influências nos níveis críticos em solo e planta, em função da localização de $\mathrm{P}$, realizou-se um ensaio em casa de vegetação, em diferentes solos de Minas Gerais.

\section{Material e mÉtodos}

Amostras de um Latossolo Vermelho Amarelo variação Una, textura muito argilosa, foram realizadas no município de Viçosa, MG, coletadas na camada de 0,10 a $0,40 \mathrm{~m}$ de profundidade, e de um Argissolo Vermelho Amarelo, textura média, do município de Ponte Nova, MG, coletadas em 0,20 m superficial. Os solos foram escolhidos em função de seus diferentes teores de argila e doravante serão denominados LU e PVA, respectivamente. As amostras foram secadas ao ar e passadas em peneira com abertura de $4 \mathrm{~mm}$ de malha, retirando-se subamostras para caracterização (Tabela 1).

Tabela 1. Características químicas e físicas das amostras de solo

\begin{tabular}{|c|c|c|}
\hline Característica & LU & PVA \\
\hline $\mathrm{pH}-\mathrm{H}_{2} \mathrm{O}(1: 2,5)$ & 4,9 & 4,9 \\
\hline $\mathrm{Al}^{3+}\left(\mathrm{cmol}_{\mathrm{c}} \mathrm{dm}^{-3}\right)^{\underline{1} /}$ & 0,8 & 0,4 \\
\hline $\mathrm{Ca}^{2+}\left(\mathrm{cmol}_{\mathrm{c}} \mathrm{dm}^{-3}\right)^{\underline{1} /}$ & 0,5 & 0,5 \\
\hline $\mathrm{Mg}^{2+}\left(\mathrm{cmol}_{\mathrm{c}} \mathrm{dm}^{-3}\right)^{\frac{1}{1}}$ & 0,0 & 0,2 \\
\hline $\mathrm{H}+\mathrm{Al}\left(\mathrm{cmol}_{\mathrm{c}} \mathrm{dm}^{-3}\right)^{2}$ & 6,4 & 8,1 \\
\hline $\mathrm{K}\left(\mathrm{mg} \mathrm{dm}^{-3}\right)^{3 /}$ & 30 & 31 \\
\hline $\mathrm{Zn}\left(\mathrm{mg} \mathrm{dm}^{-3}\right)^{3 /}$ & 0,23 & 0,75 \\
\hline$P\left(\mathrm{mg} \mathrm{dm}^{-3}\right)^{3 /}$ & 1,1 & 2,8 \\
\hline$P$ remanescente $\left(\mathrm{mg} \mathrm{L}^{-1}\right)^{4 /}$ & 9,75 & 31,1 \\
\hline Carbono orgânico (dag kg $\left.{ }^{-1}\right)^{5 /}$ & 14,1 & 8,8 \\
\hline Areia grossa $\left(\mathrm{g} \mathrm{kg}^{-1}\right)^{6}$ & 150 & 400 \\
\hline Areia fina $\left(\mathrm{g} \mathrm{kg}^{-1}\right)^{6 /}$ & 80 & 210 \\
\hline Silte $\left(\mathrm{g} \mathrm{kg}^{-1}\right) 6 /$ & 70 & 60 \\
\hline Argila $\left(\mathrm{g} \mathrm{kg}^{-1}\right)^{6}$ & 700 & 330 \\
\hline
\end{tabular}

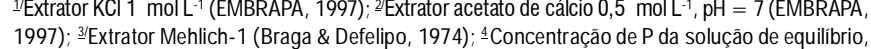
após se agitar por $1 \mathrm{~h}$ o solo com CaCl $20,01 \mathrm{~mol} \mathrm{~L}^{-1}$, contendo $60 \mathrm{mg} \mathrm{L}^{-1}$ de P. Relação 1:10 (Alvarez V. et al., 2000); ${ }^{5}$ Método de Walkley e Black (J ackson, 1958); 6/Método da pipeta (EMBRAPA, 1997)

O Zn disponível foi determinado por espectrofotometria de absorção atômica, com extrator Mehlich-1. A relação solo:solução foi de 1:10 e os períodos de agitação e repouso foram de 5 min e 16 h, respectivamente (Braga \& Defelipo, 1974).

$\mathrm{O} P$ remanescente é a concentração de $\mathrm{P}$ na solução de equilíbrio, após se agitar, por 1 hora, terra fina secada ao ar com solução de $\mathrm{CaCl}_{2}$ 0,01 mol L-1, contendo $60 \mathrm{mg} \mathrm{L}^{-1}$ de P (Alvarez V. et al., 2000). Utilizou-se uma relação solo:solução de 1:10, determinando-se o P por colorimetria, após formação do complexo fosfomolibídico reduzido (Braga \& Defelipo, 1974).

A necessidade de calagem foi estimada com base na proposta da CFSEMG (1999). Os valores de calcário, PRNT $=100$, foram de 3,54 e 1,69 tha-1 $\left(1,770\right.$ e 0,844 $\left.\mathrm{g} \mathrm{dm}^{-3}\right)$, para o LU e PVA, respectivamente.

Os tratamentos corresponderam a um fatorial completo (2 x 3 x 6), formado pela combinação de dois solos (LU e PVA), 
três localizações de $\mathrm{Pe}$ seis doses de $\mathrm{Zn}$. A unidade experimental foi constituída de um vaso que continha $3 \mathrm{dm}^{3}$ de solo, com quatro plantas por vaso. Os tratamentos foram dispostos em delineamento em blocos casualizados, com três repetições.

As amostras de solo foram incubadas durante 60 dias com calcário em condições de aerobiose; a seguir, receberam adubação de $\mathrm{K}$ na dose de $150 \mathrm{mg} \mathrm{dm}^{-3}$, menos o teor de $\mathrm{K}$ disponível, na forma de $\mathrm{K}_{2} \mathrm{SO}_{4}$; também se lhes adicionaram as seis doses de $\mathrm{Zn}$, que corresponderam a $0,1,2,4,6$ e $8 \mathrm{mg} \mathrm{dm}^{-3}$, incorporadas ao volume total de solo do vaso, na forma de solução de $\mathrm{ZnSO}_{4}$, devido a sua elevada solubilidade e apresentar sulfato em sua composição; uma semana depois, foi feita amostragem do solo.

O P foi aplicado na dose de $200 \mathrm{mg} \mathrm{dm}^{-3}$, na forma de superfosfato triplo, triturado e passado em peneira de $0,25 \mathrm{~mm}$, para aumentar sua superfície específica e, consequentemente, sua reatividade no solo; a quantidade de superfosfato triplo adicionada foi calculada para a totalidade do solo do vaso e incorporada sob três localizações, concentradas em 5, 10 e $30 \%$ do volume total de solo (Figura 1); a localização foi realizada com o auxílio de um tubo de PVC, com $10 \mathrm{~cm}$ de diâmetro, a partir de 3,5 cm de profundidade em relação à superfície do solo.

Seis sementes de milho foram semeadas na parte central do vaso; logo após a emergência, deixaram-se cinco plantas e, em novo desbaste, uma semana após a emergência, deixou-se apenas as quatro plantas mais uniformes e mais bem centralizadas no vaso, com o objetivo de obter na colheita
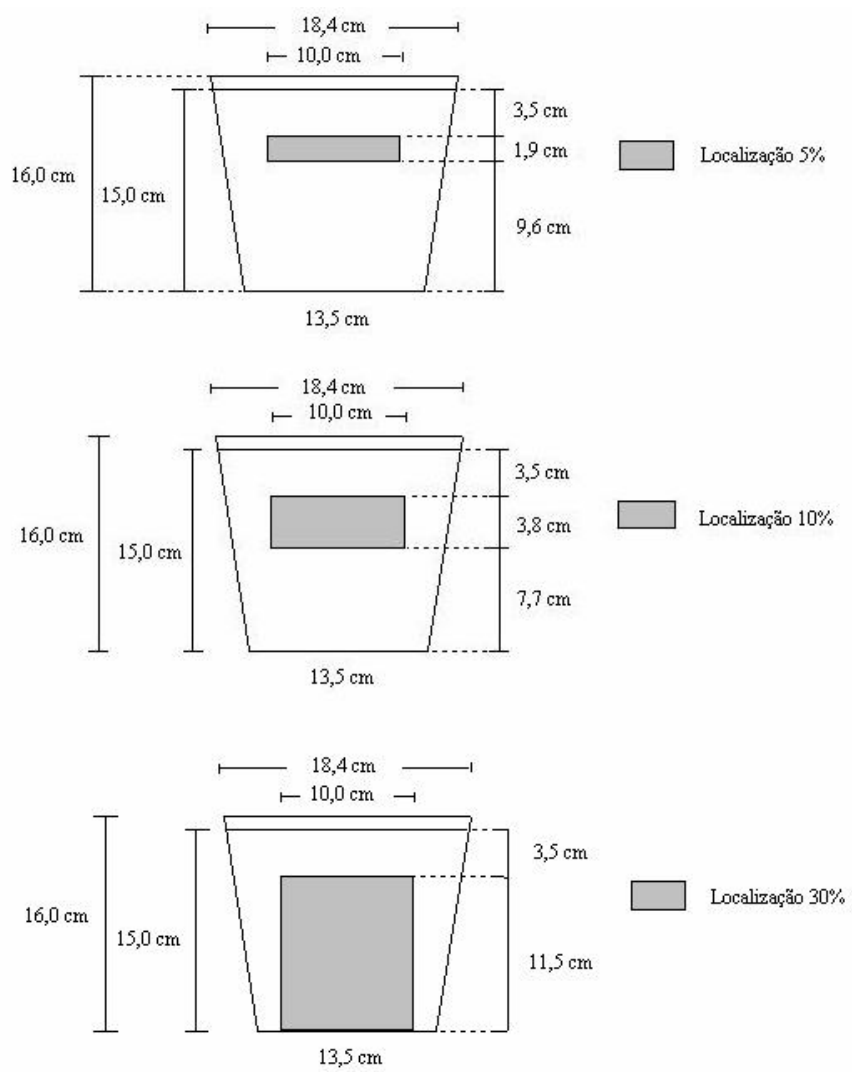

Figura 1. Corte transversal do vaso ilustrando a região de localização de P para 5\% $\left(150 \mathrm{~cm}^{3}\right), 10 \%\left(300 \mathrm{~cm}^{3}\right) \mathrm{e}$ $30 \%\left(900 \mathrm{~cm}^{3}\right)$ do volume total do solo maior massa de matéria seca e melhor avaliação dos tratamentos aplicados.

Foram realizadas quatro aplicações de solução de micronutrientes, exceto Zn (Alvarez V. et al., 1976): a primeira após o segundo desbaste e as outras uma vez por semana, até a colheita; as aplicações consistiram na adição de $0,813 \mathrm{mg}$ $\mathrm{dm}^{3} \mathrm{de} \mathrm{B}\left(\mathrm{H}_{3} \mathrm{BO}_{3}\right), 3,664 \mathrm{mg} \mathrm{dm}{ }^{3} \mathrm{de} \mathrm{Mn}\left(\mathrm{MnCl}_{2} .4 \mathrm{H}_{2} \mathrm{O}\right), 1,329 \mathrm{mg}$ $\mathrm{dm}^{3} \mathrm{de} \mathrm{Cu}\left(\mathrm{CuCl}_{2} \cdot 2 \mathrm{H}_{2} \mathrm{O}\right), 1,556 \mathrm{mg} \mathrm{dm}^{3} \mathrm{de} \mathrm{Fe}\left(\mathrm{FeSO}_{4} \cdot 7 \mathrm{H}_{2} \mathrm{O}\right) \mathrm{e}$ $0,15 \mathrm{mg} \mathrm{dm}^{3}$ de $\left.\mathrm{Mo}\left[\left(\mathrm{NH}_{4}\right)_{6} \mathrm{Mo}_{7} \mathrm{O}_{24} \cdot 4 \mathrm{H}_{2} \mathrm{O}\right)\right]$. Juntamente com a primeira aplicação de micronutrientes se incorporaram $25 \mathrm{mg}$ $\mathrm{dm}^{-3}$ de $\mathrm{N}$ (uréia) e, subsequentemente, em intervalos de sete dias, três doses de $50 \mathrm{mg} \mathrm{dm}^{-3}$ de $\mathrm{N}$ (uréia), totalizando $175 \mathrm{mg}$ $\mathrm{dm}^{-3} \mathrm{de} \mathrm{N}$, em cobertura, por unidade experimental.

Aos trinta e cinco dias após a emergência cortou-se a parte aérea das plantas de milho, que foi secada em estufa de ventilação forçada a $70{ }^{\circ} \mathrm{C}$, pesada e moída, para posterior análise; a seguir e com auxílio de um pequeno trado com diâmetro de $2 \mathrm{~cm}$ retirou-se, no centro de cada vaso e em toda a profundidade, uma amostra de solo de aproximadamente $20 \mathrm{~cm}^{3}$, para posterior análise e se determinaram, nos solos amostrados, o Zn e o P disponíveis.

O material vegetal foi submetido a digestão nitroperclórica e no extrato realizou-se a dosagem de $\mathrm{Zn}$ e P; com a massa da matéria seca e o teor de Zn, calculou-se o conteúdo de Zn na parte aérea das plantas de milho.

Os dados de solo e de planta foram submetidos à análise de variância, utilizando-se contrastes ortogonais para as comparações entre solos e localização de P no solo. Modelos de regressão foram ajustados, relacionando-se as variáveis dependentes com as doses de $\mathrm{Zn}$, escolhendo-se aqueles que, além de possuírem coeficientes de regressão significativos até o nível de $10 \%$ de probabilidade, apresentaram o maior coeficiente de determinação ajustado.

Com base nos modelos de regressão selecionados para produção de matéria seca em função das doses de $\mathrm{Zn}$, calcularam-se as doses necessárias para atingir $90 \%$ da produção máxima (doses recomendáveis). Pela substituição das "doses recomendáveis" nas equações de regressão que relacionavam os teores de $\mathrm{Zn}$ no solo e na planta com as doses adicionadas, obtiveram-se os níveis críticos deste nutriente, no solo e na planta. Finalmente se calculou, também, o conteúdo de $\mathrm{Zn}$ correspondente ao nível crítico, substituindo-se as doses recomendáveis nas equações de regressão que relacionam o conteúdo de $\mathrm{Zn}$ da parte aérea das plantas com as doses adicionadas. A eficiência de utilização de $\mathrm{Zn}$ foi calculada pelo quociente entre a matéria seca e o teor de $\mathrm{Zn}$ da parte aérea das plantas de milho, determinado no nível crítico.

\section{RESULTADOS E DISCUSSÃO}

A caracterização das amostras de solo retiradas após a incorporação dos corretivos de acidez e das doses de Zn e antes da localização do P, mostrou que o PVA apresentou maior teor de $\mathrm{Zn}$ que o LU, como reflexo da mais alta disponibilidade inicial (Tabela 1), porém a resposta à incorporação de Zn foi similar nos dois solos, como indicado pela semelhança dos valores das declividades das equações de regressão linear, relacionando-se o Zn disponível com o Zn aplicado (Tabela 2). 
Tabela 2. Zinco disponível do solo, antes e após a localização de fósforo (LP), e respectivas equações de regressão do zinco disponível em função das doses de zinco aplicadas

\begin{tabular}{|c|c|c|c|c|c|c|c|c|c|}
\hline \multirow{3}{*}{ Solo } & \multirow{3}{*}{$\begin{array}{l}\text { LP } \\
\%\end{array}$} & \multicolumn{6}{|c|}{ Doses de $\mathrm{Zn}\left(\mathrm{mg} \mathrm{dm}^{-3}\right)$} & \multirow{3}{*}{ Equação de regressão } & \multirow{3}{*}{$\mathbf{R}^{2}$} \\
\hline & & 0 & 1 & 2 & 4 & 6 & 8 & & \\
\hline & & \multicolumn{6}{|c|}{$\mathrm{mg} \mathrm{dm}^{-3}$} & & \\
\hline \multicolumn{10}{|c|}{ Antes da LP } \\
\hline LU & - & 0,25 & 0,83 & 1,70 & 3,15 & 4,91 & 5,94 & $\hat{y}=0,224+0,737^{* *} x$ & 0,996 \\
\hline PVA & - & 0,67 & 1,34 & 2,07 & 3,79 & 5,11 & 6,19 & $\hat{y}=0,710+0,710^{* *} x$ & 0,993 \\
\hline \multicolumn{10}{|c|}{ Após a LP } \\
\hline \multirow{4}{*}{ LU } & 5 & 0,72 & 1,40 & 2,32 & 3,50 & 5,01 & 6,25 & $\hat{y}=0,780+0,691^{* *} x$ & 0,998 \\
\hline & 10 & 0,53 & 1,42 & 2,08 & 3,92 & 5,00 & 5,72 & $\hat{y}=0,780+0,667^{* *} x$ & 0,977 \\
\hline & 30 & 0,47 & 1,35 & 1,18 & 3,42 & 4,85 & 6,19 & $\hat{y}=0,540+0,712^{* *} x$ & 0,999 \\
\hline & 5 & 1,32 & 2,09 & 3,08 & 4,72 & 5,27 & 7,20 & $\hat{y}=1,480+0,705^{* *} x$ & 0,981 \\
\hline \multirow[t]{2}{*}{ PVA } & 10 & 1,20 & 2,15 & 3,59 & 4,67 & 5,63 & 6,89 & $\hat{y}=1,630+0,684^{* * *} x$ & 0,969 \\
\hline & 30 & 1,26 & 1,93 & 2,89 & 4,58 & 5,42 & 7,06 & $\hat{y}=1,350+0,717^{* *} x$ & 0,991 \\
\hline
\end{tabular}

** Significativo a $1 \%$ pelo teste $\mathrm{t}$

Após a localização de $\mathrm{P}$, o teor de $\mathrm{Zn}$ no solo manteve as mesmas tendências observadas antes da localização, com o PVA apresentando valores maiores que o LU (Tabela 2), possivelmente pelo fato do $\mathrm{P}$ não ter sido misturado ao volume do solo do vaso diminuindo, assim, a possibilidade de interagir com o Zn. Haldar \& Mandal (1981) aplicaram, em todo o volume de solo do vaso, 50 e $100 \mathrm{mg} \mathrm{dm}^{-3}$ de $\mathrm{P}$, como $\mathrm{KH}_{2} \mathrm{PO}_{4}$, e 5 e $10 \mathrm{mg} \mathrm{dm}^{-3} \mathrm{de} \mathrm{Zn}$, usando como fonte o $\mathrm{ZnSO}_{4}$ e observaram interações $\mathrm{P}-\mathrm{Zn}$ em plantas de arroz atribuindo-as à mudança na disponibilidade desses elementos no solo, pela aplicação conjunta de $\mathrm{Pe} \mathrm{Zn}$.

O Zn recuperado pelo extrator Mehlich-1 relacionou-se linearmente com o Zn adicionado ao solo (Tabela 2). Os dois solos apresentaram, porém, similaridade na declividade das equações, mesmo após a localização de $\mathrm{P}$, sugerindo uma semelhança entre os solos quanto à capacidade tampão de Zn. Apesar da diferença textural, o solo PVA apresentou praticamente a mesma capacidade de troca catiônica que o LU (Tabela 1), sugerindo presença de argilas de maior atividade no seu complexo coloidal.

A produção de matéria seca respondeu à aplicação de $\mathrm{Zn}$ no LU, com diferenciação maior entre a testemunha e as doses subsequentes de Zn (Tabela 3); indicando a necessidade de se utilizar, em futuros trabalhos, doses menores de $\mathrm{Zn}$; no solo PVA não se observou resposta ao $\mathrm{Zn}$, sugerindo que os teores de Zn disponível originalmente encontrados (Tabela 1), atenderam ao requerido pelas plantas, para esta escala de produção (Tabela 3).

O milho crescido no LU respondeu à localização do $\mathrm{P}$, com maiores produções em resposta a uma localização maior do elemento (Tabela 3), em concordância com os resultados reportados por Silva et al. (2004) e Corrêa et al. (2005). Esta resposta verificada no material mais pobre em $\mathrm{Pe}$ mais argiloso, não foi evidenciada no PVA, de textura média e com maior disponibilidade inicial do nutriente (Tabela 1 e 2), sugerindo que o método de aplicação assumiria menor importância em solos com elevada disponibilidade de P. Os solos semelhantes ao PVA, com menor fator capacidade, possibilitam melhor aproveitamento do $\mathrm{P}$ pelas plantas, quando a água não é fator limitante à produção (Costa et al., 2006; Farias et al., 2008).

$\mathrm{O}$ teor de $\mathrm{Zn}$ na parte aérea aumentou linearmente com as doses (Tabela 4); contudo, esses aumentos nem sempre foram acompanhados por incrementos equivalentes na produção de matéria seca, como no PVA (Tabela 3), indicando que o Zn não chegou a ser o nutriente limitante neste solo. Santos et al. (2009) obtiveram resultados semelhantes, ou seja, os autores

Tabela 3. Produção de matéria seca da parte aérea das plantas de milho e respectivas equações de regressão, em função das doses de zinco aplicadas, considerando-se a localização de fósforo nos solos (LP)

\begin{tabular}{|c|c|c|c|c|c|c|c|c|c|}
\hline \multirow{3}{*}{ Solo } & \multirow{3}{*}{$\begin{array}{l}\mathbf{L P} \\
\%\end{array}$} & \multicolumn{6}{|c|}{ Doses de $\mathrm{Zn}\left(\mathrm{mg} \mathrm{dm} \mathrm{m}^{-3}\right)$} & \multirow{3}{*}{ Equação de regressão } & \multirow{3}{*}{$\mathbf{R}^{2}$} \\
\hline & & 0 & 1 & 2 & 4 & 6 & 8 & & \\
\hline & & \multicolumn{6}{|c|}{ g vaso $^{-1}$} & & \\
\hline \multirow{3}{*}{ LU } & 5 & 9,67 & 21,57 & 24,71 & 23,01 & 25,14 & 26,35 & $\hat{y}=10,20+13,228^{* *} x^{0,5}-2,807^{*} x$ & 0,943 \\
\hline & 10 & 11,69 & 21,28 & 20,77 & 19,47 & 21,73 & 22,00 & $\hat{y}=12,39+8,749^{* *} x^{0,5}-2,009^{*} x$ & 0,848 \\
\hline & 30 & 14,77 & 17,69 & 19,55 & 20,75 & 20,09 & 17,06 & $\hat{y}=14,35+6,235^{* *} x^{0,5}-1,743^{*} x$ & 0,835 \\
\hline \multirow{3}{*}{ PVA } & 5 & 22,83 & 22,67 & 24,05 & 20,53 & 25,73 & 19,91 & $\hat{y}=\bar{y}=22,62$ & - \\
\hline & 10 & 23,15 & 25,24 & 20,74 & 25,17 & 21,20 & 21,91 & $\hat{y}=\bar{y}=22,90$ & - \\
\hline & 30 & 24,01 & 23,36 & 22,63 & 21,61 & 26,14 & 19,01 & $\hat{y}=\bar{y}=22,79$ & - \\
\hline
\end{tabular}


Tabela 4. Teor e conteúdo de zinco da parte aérea das plantas de milho e respectivas equações de regressão, em função das doses de zinco, considerando-se a localização de fósforo nos solos (LP)

\begin{tabular}{|c|c|c|c|c|c|c|c|c|c|}
\hline \multirow{2}{*}{ Solo } & \multirow{2}{*}{$\begin{array}{l}\text { LP } \\
\%\end{array}$} & \multicolumn{6}{|c|}{ Doses de $\mathrm{Zn}\left(\mathrm{mg} \mathrm{dm}^{-3}\right)$} & \multirow{2}{*}{ Equação de regressão } & \multirow{2}{*}{$\mathbf{R}^{2}$} \\
\hline & & 0 & 1 & 2 & 4 & 6 & 8 & & \\
\hline \multicolumn{10}{|c|}{ Teor de $\mathrm{Zn}\left(\mathrm{mg} \mathrm{kg}^{-1}\right)$} \\
\hline \multirow{4}{*}{ LU } & 5 & 20,4 & 16,3 & 26,4 & 33,4 & 35,8 & 49,3 & $\hat{y}=17,3+13,716^{* *} x$ & 0,920 \\
\hline & 10 & 14,6 & 15,7 & 21,1 & 30,6 & 40,6 & 50,4 & $\hat{y}=12,5+4,664^{* *} x$ & 0,992 \\
\hline & 30 & 16,8 & 20,2 & 28,2 & 41,7 & 64,2 & 63,8 & $\hat{y}=15,8+4,145^{* *} x$ & 0,952 \\
\hline & 5 & 30,3 & 45,6 & 31,8 & 42,8 & 46,8 & 52,3 & $\hat{y}=33,8+2,227^{* *} x$ & 0,616 \\
\hline \multirow[t]{4}{*}{ PVA } & 10 & 25,0 & 25,3 & 40,9 & 36,3 & 68,7 & 57,1 & $\hat{y}=24,9+4,945^{* *} x$ & 0,753 \\
\hline & 30 & 17,5 & 26,3 & 36,4 & 44,6 & 49,1 & 60,2 & $\hat{y}=21,8+4,934^{* *} x$ & 0,953 \\
\hline & \multicolumn{9}{|c|}{ Conteúdo de Zn ( $\mu \mathrm{g}$ vaso $\left.{ }^{-1}\right)$} \\
\hline & 5 & 197 & 341 & 635 & 765 & 892 & 1.291 & $\hat{y}=250+125^{* *} x$ & 0,954 \\
\hline \multirow[t]{3}{*}{ LU } & 10 & 164 & 330 & 437 & 588 & 869 & 1.079 & $\hat{y}=\bar{y}=578$ & - \\
\hline & 30 & 253 & 357 & 524 & 857 & 1.264 & 965 & $\hat{y}=\bar{y}=703$ & - \\
\hline & 5 & 708 & 1.077 & 750 & 873 & 1.196 & 1.018 & $\hat{y}=\bar{y}=937$ & - \\
\hline \multirow[t]{2}{*}{ PVA } & 10 & 526 & 622 & 846 & 903 & 1.486 & 1.236 & $\hat{y}=\bar{y}=936$ & - \\
\hline & 30 & 422 & 611 & 823 & 960 & 1.292 & 1.169 & $\hat{y}=\bar{y}=880$ & - \\
\hline
\end{tabular}

$\#$, ** Significativo a 10 e $1 \%$, respectivamente, pelo teste $\mathrm{t}$

constataram que mesmo com o aumento na concentração de Zn nas plantas de sorgo em função da aplicação de doses crescentes de $\mathrm{Zn}$, não houve resposta na produção de grãos.

As plantas cultivadas no PVA apresentaram maior concentração de Zn que aquelas cultivadas no LU (Tabela 4), em resposta a uma disponibilidade maior de $\mathrm{Zn}$ neste solo (Tabela 1).

As localizações de $\mathrm{P}$ nos menores volumes de solo (5 e $10 \%$ ) levaram a menores concentrações de $\mathrm{Zn}$ na parte aérea das plantas de milho cultivadas no LU, quando comparadas com a localização em 30\% (Tabela 4). Paralelamente, a maior produção de matéria seca com o $\mathrm{P}$ mais localizado nos menores volumes de solo (Tabela 3) provocou uma diluição do Zn no tecido vegetal. Como a concentração de $Z$ n e a produção de matéria seca apresentaram comportamentos antagônicos, dependendo da localização do $\mathrm{P}$, o conteúdo de $\mathrm{Zn}$, que é resultante do produto dessas duas variáveis, não foi alterado (Tabela 4).
No PVA, onde não houve efeito de localização sobre a produção de matéria seca da parte aérea (Tabela 3 ), o conteúdo de Zn não dependeu da localização de P (Tabela 4), pela maior disponibilidade inicial deste nutriente (Tabela 1).

$\mathrm{O}$ conteúdo de $\mathrm{Zn}$ da parte aérea das plantas de milho aumentou com as doses de $\mathrm{Zn}$ independe do solo (Tabela 4); no solo LU este incremento se deveu ao aumento na maior concentração de Zn e à maior produção de matéria seca; no solo PVA ele foi devido apenas ao aumento na concentração de Zn.

A concentração de $\mathrm{P}$ na parte aérea das plantas apresentou efeito significativo quanto à sua localização (Tabela 5). No solo mais argiloso (LU), as plantas apresentaram maior teor de P na parte aérea para as localizações em 5 e $10 \%$ do volume total do solo. Castilhos \& Anghinoni (1983) constataram que a aplicação do fertilizante fosfatado a uma fração menor de um volume de solo, teve a vantagem de proteger o $\mathrm{P}$ contra as reações de adsorção. Para uma mesma dose de $\mathrm{P}$, quanto menor

Tabela 5. Teor de fósforo da parte aérea das plantas de milho e respectivas equações de regressão, em função das doses de zinco, considerando-se a localização de fósforo nos solos (LP)

\begin{tabular}{|c|c|c|c|c|c|c|c|c|c|}
\hline \multirow{3}{*}{ Solo } & \multirow{3}{*}{$\begin{array}{l}\text { LP } \\
\%\end{array}$} & \multicolumn{6}{|c|}{ Doses de $\mathrm{Zn}\left(\mathrm{mg} \mathrm{dm} \mathrm{m}^{-3}\right)$} & \multirow{3}{*}{ Equação de regressão } & \multirow{3}{*}{$R^{2}$} \\
\hline & & 0 & 1 & 2 & 4 & 6 & 8 & & \\
\hline & & \multicolumn{6}{|c|}{$\mathrm{g} \mathrm{kg}^{-1}$} & & \\
\hline \multirow{3}{*}{ LU } & 5 & 8,6 & 3,0 & 2,3 & 2,1 & 2,1 & 2,5 & $\hat{y}=8,4-6,61^{* *} x^{0,5}+1,63^{* *} x$ & 0,988 \\
\hline & 10 & 4,4 & 2,5 & 2,5 & 2,2 & 2,3 & 2,2 & $\hat{y}=4,3-1,99^{* *} x^{0,5}+0,452^{* * *} x$ & 0,970 \\
\hline & 30 & 3,2 & 2,2 & 1,8 & 2,3 & 2,0 & 1,9 & $\hat{y}=3,1-1,08^{* *} x^{0,5}+0,247^{* *} x$ & 0,825 \\
\hline \multirow{3}{*}{ PVA } & 5 & 2,6 & 2,6 & 2,6 & 3,2 & 2,4 & 2,7 & $\hat{y}=\bar{y}=2,7$ & - \\
\hline & 10 & 3,2 & 2,8 & 2,6 & 2,5 & 3,1 & 2,8 & $\hat{y}=\bar{y}=2,8$ & - \\
\hline & 30 & 2,9 & 3,0 & 3,1 & 2,8 & 2,4 & 2,9 & $\hat{y}=\bar{y}=2,9$ & - \\
\hline
\end{tabular}


a fração do volume de solo fertilizado maior será a dose relativa e, portanto, maior também a disponibilidade de $\mathrm{P}$ nesta região; quanto ao solo PVA, por ser menos argiloso e ter maior disponibilidade de $\mathrm{P}$, não se observou efeito de localização.

A diminuição do teor de $\mathrm{P}$ com as doses de Zn no LU ocorreu concomitante com o incremento da matéria seca da parte aérea, em resposta à aplicação de Zn neste solo, observando-se efeito de diluição (Tabela 3 e 5); isto, porém, não se verificou no PVA, em que não houve alterações na produção de matéria seca com as doses de $\mathrm{Zn}$.

Para a dose zero de Zn no solo LU e na localização 5\% de P, observou-se teor elevado de $\mathrm{P}$ na parte aérea das plantas (Tabela 5), com diminuição na produção de matéria seca (Tabela 3); segundo Pinton et al. (1993) a deficiência de Zn aumenta a permeabilidade da plasmalema das células das raízes, favorecendo a absorção de P. Assim, uma absorção maior de P em plantas deficientes em Zn pode, em parte, ser expressão de uma permeabilidade maior das células das raízes ou de uma desregulação do controle da carga para o xilema. No entanto, neste trabalho o teor de $\mathrm{Zn}$ observado na parte aérea das plantas de milho crescidas no LU, na maior localização de P e na dose zero de $\mathrm{Zn}$, não difere acentuadamente daqueles determinados para os outros tratamentos (Tabela 4).

Por outro lado, quando a concentração de $\mathrm{P}$ nas plantas está excepcionalmente elevada, tendendo à toxidez, é comum o aparecimento de sintomas de deficiência de $\mathrm{Zn}$ induzida por P. Cakmak \& Marschner (1987) verificaram que, com o aumento dos teores de P na parte aérea, os sintomas de deficiência de $\mathrm{Zn}$ se tornaram mais severos, apesar do teor de $\mathrm{Zn}$ não decrescer. Isto se pode explicar por uma diminuição na disponibilidade fisiológica de Zn, ou seja, redução de sua solubilidade e mobilidade causada por uma precipitação de $\mathrm{Zn}$ na forma de fosfato de zinco, devida aos altos teores de P na parte aérea. Com isto, os teores totais de Zn não se alteram porém a atividade do $\mathrm{Zn}$ fica comprometida.

O solo PVA, por conter maior teor de Zn (Tabela 1), possibilitou maior absorção desse nutriente pela planta, na dose zero do elemento (Tabela 4), embora não se tenha observado teores elevados de $\mathrm{P}$ na parte aérea das plantas (Tabela 5).

Os valores de produção de matéria seca, teor e conteúdo de $\mathrm{Zn}$ e teor de $\mathrm{P}$ da parte aérea (Tabelas 3, 4 e 5) sugerem que ocorre uma relação $\mathrm{Zn} / \mathrm{P}$ ideal para o crescimento das plantas. Valores inferiores a 3,2, expressando os teores em mg kg-1 de $\mathrm{Zn} \mathrm{e} \mathrm{g} \mathrm{kg}^{-1}$ de $\mathrm{P}$, respectivamente, prejudicariam sensivelmente a produção de matéria seca; este foi o menor valor achado para o quociente, neste trabalho, em plantas normais, crescidas na dose zero de Zn. Comparando-se nesta dose de Zn, a localização $5 \%$ com as de 10 e $30 \%$ no solo LU, observa-se tendência de maior produção de matéria seca para as plantas crescidas nos tratamentos com menores localizações (10 e 30\%); o menor teor de $\mathrm{P}$ nas folhas aumentou a relação $\mathrm{Zn} / \mathrm{P}$, favorecendo o crescimento das plantas.

Quando comparados os tratamentos do LU com os do PVA na dose zero de $\mathrm{Zn}$, eram esperadas maiores interações P-Zn em plantas cultivadas no PVA, em virtude da maior disponibilidade de P. Costa et al. (2006) indicam que solos menos argilosos possibilitam um aproveitamento melhor do $\mathrm{P}$ disponível, quando a água não é fator limitante à produção, mas no PVA os teores de $\mathrm{Zn}$, tanto no solo quanto na planta, foram maiores que no LU, proporcionando melhor balanço na relação $\mathrm{Zn} / \mathrm{P}$, favorecendo o crescimento das plantas, cuja tendência parece reforçar a importância do papel do Zn na seletividade da absorção de P.

Estimaram-se os níveis críticos de Zn no solo e nas plantas de milho. Inicialmente, com base nos modelos de regressão selecionados para a produção de matéria seca em função das doses de $\mathrm{Zn}$ aplicadas (Tabela 3), foram calculadas as doses necessárias para atingir a produção máxima e a de $90 \%$ desse valor (doses recomendáveis). Dos modelos testados, o quadrático base raiz quadrada foi o que melhor se ajustou aos dados (Tabela 3); pela substituição dessas doses recomendáveis nas equações que relacionam os teores de $\mathrm{Zn}$ do solo recuperados pelo extrator Mehlich-1 com o Zn aplicado (Tabela 2), estimou-se o nível crítico do nutriente no solo; substituindo-se as doses recomendadas nas equações que relacionam a concentração de $\mathrm{Zn}$ na parte aérea das plantas com as doses adicionadas (Tabela 4), determinou-se o nível crítico do nutriente na planta, em que esses valores, acrescidos do conteúdo de $\mathrm{Zn}$ no vegetal e da eficiência de utilização de Zn, calculada pela relação entre a produção de matéria seca e o teor do nutriente na parte aérea, são apresentados na Tabela 6 .

O nível crítico de Zn no solo variou para o LU, entre 0,91 e 2,13 mg dm${ }^{-3}$; nas plantas, o intervalo foi de 18,0 a 24,6 $\mathrm{mg} \mathrm{kg}^{-1}$; referidas variações refletem a interferência das características dos solos e dos tratamentos efetuados sobre os valores observados. Galrão (1995) obteve níveis críticos de Zn no solo (Mehlich-1) de 0,7 mg dm${ }^{-3}$ e de 18,5 $\mathrm{mg} \mathrm{kg}^{-1}$ nas folhas de milho cultivado em um LV, em condições de campo.

Para o solo LU, quanto maior a localização de P, maiores os níveis críticos de $\mathrm{Zn}$ no solo e na parte aérea das plantas de milho (Tabela 6). No tratamento de maior localização (5\%), haveria um estímulo ao crescimento radicular na parte fertilizada com $\mathrm{P}$, concentrando raízes nesta região; por outro lado, na localização de $30 \%$ as raízes ocupariam, de forma mais homogênea, o volume do solo no vaso.

Assim, como o $\mathrm{Zn}$ foi distribuído quase que na totalidade da amostra de solo e sendo um nutriente transportado por difusão, a maior exploração do sistema radicular levaria a uma concentração de Zn menor no nível crítico.

No solo PVA, como não foi possível ajustar regressões da produção de matéria seca e do teor de Zn da parte aérea das plantas de milho em função das doses de $\mathrm{Zn}$ aplicadas (Tabela 3 e 4), não foram obtidas as doses para máxima produção de matéria seca, fazendo com que os níveis críticos sejam representados pelas médias em cada localização de $\mathrm{P}$ (Tabela 6). Mesmo assim se observou no solo PVA, comportamento semelhante dos níveis críticos no solo e na planta, ao que ocorreu com o solo LU, com maiores níveis críticos de Zn para as localizações 5 e $10 \%$ de $\mathrm{P}$ (Tabela 6).

A eficiência da utilização de Zn na dose recomendável aumentou quando o $\mathrm{P}$ foi menos localizado (10 e 30\%) (Tabela 6); a maior localização de $\mathrm{P}$, estimulando maior concentração de raízes em menor volume de solo, resultou em maior nível crítico de Zn, o que indicaria menor eficiência de utilização, porém praticamente não houve alteração na eficiência de 
Tabela 6. Produção máxima de matéria seca da parte aérea de plantas de milho, 0,9 da produção máxima (Y), doses de zinco recomendáveis para atingi-las $\left(\mathrm{Zn}_{\text {DosE }}\right)$ e valores de zinco no nível crítico, considerando-se a localização de fósforo nos solos (LP)

\begin{tabular}{|c|c|c|c|c|c|c|c|c|c|}
\hline \multirow{3}{*}{ Solo } & \multirow{3}{*}{$\begin{array}{l}\text { LP } \\
\%\end{array}$} & \multicolumn{4}{|c|}{ Produção } & \multirow{2}{*}{\multicolumn{4}{|c|}{ Nível Crítico ${ }^{\underline{1} /}$}} \\
\hline & & \multicolumn{2}{|c|}{ Máxima } & \multicolumn{2}{|c|}{ 0,9 Máxima } & & & & \\
\hline & & $\begin{array}{c}\text { Y } \\
\text { g vaso-1 }^{-1}\end{array}$ & $\begin{array}{c}\mathrm{Zn}_{\text {DOSE }} \\
\mathrm{mg} \mathrm{dm}^{-3}\end{array}$ & $\begin{array}{c}Y \\
\text { g vaso-1 }^{-1}\end{array}$ & $\begin{array}{c}\mathrm{Zn}_{\text {DOSE }} \\
\mathrm{mg} \mathrm{dm}^{-3}\end{array}$ & $\begin{array}{c}\mathrm{Zn}_{\mathrm{SOLO}} \\
\mathrm{mg} \mathrm{dm}^{-3}\end{array}$ & $\begin{array}{l}\mathrm{Zn}_{\text {PLANTA }} \\
\mathrm{mg} \mathrm{kg}^{-1}\end{array}$ & $\begin{array}{c}\mathrm{Zn}_{\text {CONT. }} \\
\mu \mathrm{g} \text { vaso- }^{-1}\end{array}$ & $\begin{array}{c}\mathrm{Zn}_{\mathrm{EU}} \\
\mathrm{g}^{2} \mu \mathrm{g}^{-1}\end{array}$ \\
\hline \multirow{3}{*}{ LU } & 5 & 25,78 & 5,55 & 23,20 & 1,96 & 2,13 & 24,6 & 495 & 0,94 \\
\hline & 10 & 21,91 & 4,74 & 19,72 & 1,28 & 1,63 & 18,5 & 331 & 1,07 \\
\hline & 30 & 19,92 & 3,20 & 17,93 & 0,52 & 0,91 & 18,0 & 367 & 0,99 \\
\hline \multirow[t]{2}{*}{ PVA } & 10 & 22,90 & 0 & 20,61 & 0 & $<1,63$ & $<24,9$ & $<563$ & $>0,83$ \\
\hline & 30 & 22,79 & 0 & 20,51 & 0 & $<1,35$ & $<21,8$ & $<530$ & $>0,94$ \\
\hline
\end{tabular}

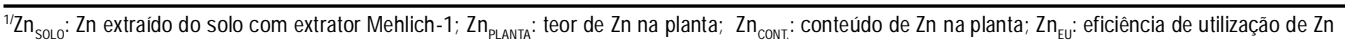

utilização, haja vista que a concentração de P levou a uma produção maior de matéria seca.

\section{CONCLUSÕES}

1. A adição de zinco aumentou linearmente o teor do nutriente no solo e a absorção pela planta.

2. No Latossolo Vermelho Amarelo variação una, os níveis críticos de zinco pelo extrator Mehlich-1 variaram de 0,91 a $2,13 \mathrm{mg} \mathrm{dm}^{-3} \mathrm{e}$, na planta, de 18,0 a $24,6 \mathrm{mg} \mathrm{kg}^{-1}$.

3. Os níveis críticos de zinco foram maiores quando as aplicações de fósforo foram localizadas em menor volume de solo.

4. Quando o solo foi fertilizado com zinco, a maior localização de fósforo proporcionou maior produção de matéria seca e decréscimo no teor de zinco na planta.

5. No Latossolo Vermelho Amarelo variação una ocorreu interação negativa da localização de fósforo e das doses de zinco.

\section{LITERATURA CITADA}

Alvarez V., V. H.; Braga, J. M.; Estevão, M. de M.; Pinto, O. C. B. Equilíbrio de formas disponíveis de fósforo e enxofre em dois Latossolos de Minas Gerais: I - Equílibrio fósforoenxofre. Experientiae, v.22, n.1, p.1-29, 1976.

Alvarez V., V. H.; Novais, R. F.; Dias, L. E.; Oliveira, J. A. Determinação e uso do fósforo remanescente. Viçosa: Sociedade Brasileira de Ciência do Solo, 2000. p.27-33. Boletim Informativo, 25.

Braga, J. M.; Defelipo, B. V. Determinação espectrofotométrica de fósforo em extrato de solo e material vegetal. Revista Ceres, v.21, p.73-85, 1974.

Cakmak, I.; Marschner, H. Mechanism of phosphorus-induced zinc deficiency in cotton. III. Changes in physiological availability of zinc in plants. Plant Physiology, v.70, p.13-20, 1987.

Carneiro, L. F.; Furtini Neto, A. E.; Rezende, A. V.; Curi, N.; Santos, J. Z. L.; Lago, F. J. Fontes, doses e modos de aplicação de fósforo na interação fósforo-zinco em milho. Ciência e Agrotecnologia, v.32, n.4, p.1133-1141, 2008.
Castilhos, D. D.; Anghinoni, I. Eficiência na absorção e disponibilidade de fósforo pelo milho em relação ao método de aplicação do fertilizante ao solo. Agronomia Sulriograndense, v.19, p.43-55, 1983.

CFSEMG - Comissão de Fertilidade do Solo do Estado de Minas Gerais. Recomendações para o uso de corretivos e fertilizantes em Minas Gerais ( $5^{\mathrm{a}}$ aproximação). Viçosa: FAPEMIG, 1999. 359p.

Corrêa, R. M.; Nascimento, C. W. A.; Souza, S. K. S. C.; Freire, F. J.; Ferraz, G. B. Gafsa rock phosphate and the triple superphosphate for dry matter production and $\mathrm{P}$ uptakeby corn. Scientia Agrícola, v.62, n.2, p.159-164, 2005.

Costa, J. P. V.; Barros, N. F.; Albuquerque, A. W.; Moura Filho, G.; Santos, J. R. Fluxo difusivo de fósforo em função de doses e da umidade do solo. Revista Brasileira de Engenharia Agrícola e Ambiental, v.10, n.4, p.828-835, 2006.

Elrashidi, M. A.; O'Connor, G. A. Influence of solution composition on sorption of zinc by soils. Soil Science Society of America Journal, v.46, p.1153-1158, 1982.

EMBRAPA - Empresa Brasileira de Pesquisa Agropecuária. Manual de métodos de análises de solo. 2.ed. Rio de Janeiro: MAB, 1997. 212p.

Farias, C. H. de A.; Fernandes, P. D.; Azevedo, H. M. de; Dantas Neto, J. Índices de crescimento da cana-de-açúcar irrigada e de sequeiro no Estado da Paraíba. Revista Brasileira de Engenharia Agrícola e Ambiental, v.12, n.4, p.356-362, 2008.

Furlani, A. M. C.; Furlani, P. R.; Meda, A. R,; Duarte, A. P. Efficiency of mayse cultivars for zinc uptake and use. Scientia Agrícola, v.62, n.3, p.264-273, 2005.

Galrão, E. Z. Níveis críticos de zinco para o milho cultivado em Latossolo Vermelho Amarelo, fase cerrado. Revista Brasileira de Ciência do Solo, v. 19, p.255-260, 1995.

Haldar, M.; Mandal, L. N. Effect of phosphorus and zinc on the growth and phosphorus, zinc, cooper, iron and manganese nutrition of rice. Plant Soil, v.59, p.415-425, 1981.

Jackson, M. L. Soil chemical analysis. New Jersey: PrenticeHall, 1958. 498p.

Loneragan, J. F.; Gruner, R. M.; Welch, E. A.; Aduayi, A.; Lazar, V. A.; Cary, E. E. Phosphorus accumulation and toxicity in leaves in relation to zinc supply. Soil Science Society of America Journal, v.46, p.345-352, 1982.

Marinho, M. L.; Igue, K. Factors affecting zinc absorption by corn from volcanic ash soils. Agronomy Journal, v.64, p.3-8, 1972. 
Pinton. R.; Cakmak, I.; Marschner, H. Effect of zinc deficiency on proton fluxes in plasma membrane-enriched vesicles isolated from bean roots. Journal of Experimental Botany, v.44, p.623-630, 1993.

Robson, A. D.; Pitman, M. G. Interactions between nutrients in higher plants. In: Lauchili, A.; Bieleski, R. L. (ed.). Inorganic plant nutrition. Berlin: Springer-Verlag, 1983. p.147-180.

Santos, H. C.; Fraga, V. S.; Raposo, R. W. C.; Pereira, W. E. Cu e Zn na cultura do sorgo cultivado em três classes de solos. I. Crescimento vegetativo e produção. Revista Brasileira de Engenharia Agrícola e Ambiental, v.13, n.2, p.125-130, 2009.
Shuman, L. M. Effect of phosphorus level on extractable micronutrients and their distribution among soil fractions. Soil Science Society of America Journal, v.52, p.136-141, 1988.

Silva, E. M. B.; Freire, F. J.; Santos, M. V. F.; Silva, T. J. A.; Freire, M. B. G. S. Níveis críticos de fósforo para Brachiaria brizantha e suas relações com características físicas e químicas em solos de Pernambuco. Revista Brasileira da Ciência do Solo, v.28, p.281-288, 2004.

Souza, C. A. S.; Corrêa, F. L. de O.; Mendonça, V.; Vichiato, M.; Carvalho, J. G. de. Doses de fósforo e zinco no acúmulo de macro e micronutrientes em mudas de cacaueiro. Agrotrópica, v.18, p.25-38, 2006. 